\title{
GENERAL MATRIX PENCIL TECHNIQUES FOR SOLVING DISCRETE-TIME NONSYMMETRIC ALGEBRAIC RICCATI EQUATIONS*
}

\author{
MARC JUNGERS ${ }^{\dagger}$, CRISTIAN OARĂ $\breve{~}^{\ddagger}$, HISHAM ABOU-KANDIL ${ }^{\S}$, AND RADU ŞTEFAN ${ }^{\ddagger}$
}

\begin{abstract}
A discrete-time nonsymmetric algebraic Riccati system which incorporates as special cases various discrete-time nonsymmetric algebraic Riccati equations is introduced and studied without any restrictive assumptions on the matrix coefficients. Necessary and sufficient existence conditions together with computable formulas for the stabilizing solution are given in terms of proper deflating subspaces of an associated matrix pencil. The theory is applied in the framework of game theory with an open-loop information structure to design Nash strategy without the classical assumptions on the invertibility of some matrix coefficients.
\end{abstract}

Key words. Discrete-time Nonsymmetric Algebraic Riccati Equations, Matrix Pencil, Game Theory, Deflating subspaces.

AMS subject classifications. 15A22, 65F05, 91A50.

1. Introduction. Starting with the seminal works of Kalman [23], Popov [39] and Willems [48], the standard algebraic Riccati equation - both in the continuous and discrete-time versions - has been widely investigated due to its prominent role played in control engineering [26, 28, 5, 41]. Algebraic Riccati equations offer simultaneously a nice theoretical support together with numerically efficient algorithms to cope with numerous applications in systems theory, optimal control, and filtering problems.

The theory of symmetric Riccati equations has been approached from several standpoints: time-domain, frequency domain, and state-space, leading to characterizations in terms of input-output operator, transfer matrix function, and Hamiltonian matrix, all associated with the underlying Hamiltonian system. Among these approaches, the method of invariant subspaces associated with Hamiltonian matrices for continuous-time (or symplectic matrices for discrete-time) play a paramount role $[25,28,29,27,40,30,49,45]$. The invariant subspace criteria for matrices have been extended to matrix pencils by the introduction of the Extended Hamiltonian Pencils in [46] while the place of the invariant subspace is taken by the proper deflating subspaces [34]. While pushing forward the algebraic Riccati techniques an interesting situation developed: the algebraic Riccati equation as such was no longer defined, and had to be replaced by an appropriate more general substitute. It turned out that the right substitute are the Riccati systems which can tackle the whole range of applications that one encounters in practice [20, 19, 21, 35].

Besides the symmetric Riccati equation, the theory of nonsymmetric algebraic Riccati equation, both continuous and discrete-time, has received recently a renewed interest due to a large number of applications in various fields, including applied mathematics, engineering and economic science (see for instance $[1,6,22,18]$ ). The nonsymmetric algebraic Riccati equation extends the concept of standard Riccati

\footnotetext{
${ }^{*}$ Manuscript received September 9, 2009

${ }^{\dagger}$ Centre de Recherche en Automatique de Nancy (CRAN), Nancy-Université, CNRS, 2 avenue de la Forêt de Haye F-54516 Vandoeuvre-les-Nancy. (marc.jungers@cran.uhp-nancy.fr)

${ }^{\ddagger}$ Faculty of Automatic Control and Computers, University Politehnica Bucharest, Splaiul Independentei 313 Romania (\{oara,stefan\}@riccati.pub.ro). Their work has been supported by the Romanian National University Research Council (CNCSIS) under grant ID 814/2007.

$\S$ SATIE, ENS Cachan, CNRS, 61 av du President Wilson, F-94230 Cachan, France (abou-kandil@satie.ens-cachan.fr)
} 
equation by allowing for a not necessarily square matrix variable. In the literature, these equations are referred alternatively as nonsymmetric, asymmetric, rectangular, or simply nonstandard Riccati equations. Again, different approaches based on the input-output operator, an associated Popov function, or invariant subspaces of an underlying characteristic matrix are possible. The Popov function based approach for nonsymmetric algebraic Riccati equation has been developed in [24, 37]. Iterative numerical methods based on Newton's method and various refinements to solve nonsymmetric algebraic Riccati equation can be found in $[4,15,16]$. The invariant subspace method has been extended under various restrictive assumptions on the matrix coefficients in $[1,9,10]$. Based on these theoretical results, several numerical solvers have been developed $[8,14]$.

The manifest aim of this paper is to extend the invariant subspace method for solving discrete-time nonsymmetric algebraic Riccati equation (DTNARE) without assuming any restrictive assumption on the matrix coefficients, and to explore their algorithmic consequences. While doing so, we will need to replace the DTNARE with a more powerful substitute - called the discrete-time nonsymmetric algebraic Riccati system (DTNARS) - which allows the full range of matrix coefficients, and the characteristic matrix with an appropriate matrix pencil.

The paper is organized as follows. Section 2 contains some general notation, definitions and basic results concerning matrix pencils. In Section 3 we introduce the basic setting for our approach to the discrete-time nonsymmetric Riccati theory. The central notion is the Popov-system with which we associate several mathematical objects that play various parts in the discrete-time nonsymmetric Riccati theory and for which we define an equivalence transformation. The main result of the paper establishing a one-to-one correspondence between stabilizing solutions to the discrete-time nonsymmetric algebraic Riccati system and stable deflating subspaces of the Popovsystem matrix pencil is provided in Section 4. In Section 5, we strengthen our main result to show that proper deflating subspaces provide the right framework for a practical numerical algorithm, and sketch a prototype algorithm for the computation of stabilizing solutions. The efficiency of our matrix pencil approach which avoids classical invertibility assumptions is illustrated in Section 6. There, we consider in detail one application from the realm of game theory in which discrete-time nonsymmetric algebraic Riccati equations with general matrix coefficients occur naturally: designing Nash strategy. Some relevant numerical examples are given in Section 7. Final conclusions are drawn in Section 8.

2. Preliminaries. In this section we introduce some notation and definitions, and review certain basic results needed in the sequel.

2.1. Basic notation. By $\mathbb{R}, \mathbb{C}$, and $\mathbb{N}$ we denote the real axis, the complex plane, and the set of nonnegative integers, respectively. Let $\mathbb{N}^{*}:=\mathbb{N}-\{0\}$. The open unit disk and its closure in the complex plane are denoted by $\mathbb{D}$ and $\overline{\mathbb{D}}$. For a constant matrix $A$ we denote by $A^{\prime}$ its transpose and if $A$ is invertible by $A^{-1}$ its inverse. $\operatorname{Im}(A)$ will stand for the image of $A$. A matrix pair $(A, B)$, with $A \in \mathbb{R}^{n \times n}$, $B \in \mathbb{R}^{n \times m}$, is called controllable if $\operatorname{rank}\left[\begin{array}{ll}z I-A & B\end{array}\right]=n$, for all $z \in \mathbb{C}$. A matrix pair $(C, A)$, with $A \in \mathbb{R}^{n \times n}, C \in \mathbb{R}^{p \times n}$, is called observable provided the pair $\left(A^{\prime}, C^{\prime}\right)$ is controllable. $I_{n_{1}}$ and $0_{n_{1} \times n_{2}}$ denote the identity matrix of size $n_{1} \times n_{1}$ and the null matrix of size $n_{1} \times n_{2}$, respectively. For the sake of simplicity, the dimension of the 
identity and null matrices will be avoided when obvious. Let

$$
\operatorname{diag}\left(A_{1}, \ldots, A_{k}\right):=\left[\begin{array}{ccc}
A_{1} & & \\
& \ddots & \\
& & A_{k}
\end{array}\right]
$$

denote a block diagonal matrix defined by the possible rectangular matrices $A_{1}, \ldots, A_{k}$. In a block matrix, $\star$ stands for an irrelevant block. Capital letters will denote constant matrices and bold capital letters will denote rational matrix functions. For a rational matrix function $\mathbf{S}(z)$ with real coefficients, let $\mathbf{S}^{*}(z):=\mathbf{S}^{\prime}\left(\frac{1}{z}\right)$ be the conjugate.

2.2. Matrix pencils and proper deflating subspaces. We remind in this subsection some definitions and properties of matrix pencils $[12,13]$ with a particular emphasis on proper deflating subspaces. Proper deflating subspaces are a technical tool that will allow to formulate an "invariant subspace" criteria to solve DTNARS and DTNARE without any assumption on the matrix coefficients.

Let $M$ and $N$ be $m \times n$ matrices with elements in $\mathbb{R}$. The first degree matrix polynomial $z M-N$ is called a matrix pencil or, briefly, pencil. The pencil is called regular if it is square $(m=n)$ and has a non-vanishing determinant, i.e., $\operatorname{det}(z M-$ $N) \not \equiv 0$. A pencil which is not regular is called singular. The normal rank of the pencil - denoted $r=\operatorname{rank}_{n}(z M-N)$ - is defined as the rank of $z M-N$ for almost all $z \in \mathbb{C}$ (but a finite number of points). For a regular pencil we have $m=n=r$. If $\nu_{\ell}:=m-r>0$ then we say the pencil has a (nontrivial) left singular structure. If $\nu_{r}:=n-r>0$ then the pencil has a (nontrivial) right singular structure.

Two pencils $z M-N$ and $z \widetilde{M}-\widetilde{N}$, with $M, N, \widetilde{M}, \widetilde{N} \in \mathbb{R}^{m \times n}$, are called strictly equivalent if there are two constant invertible matrices $Q \in \mathbb{R}^{m \times m}, Z \in \mathbb{R}^{n \times n}$, such that $Q(z M-N) Z=z \widetilde{M}-\widetilde{N}$. For any pencil $z M-N$ there exist a strict equivalence transformation (see for example [12]) that brings it to the Kronecker canonical form

$$
Q(z M-N) Z=\operatorname{diag}\left(L_{\epsilon_{1}}, \ldots, L_{\epsilon_{\nu_{r}}}, z M_{\infty}-I_{n_{\infty}}, z I_{n_{f}}-N_{f}, L_{\eta_{1}}^{T}, \ldots L_{\eta_{\nu_{\ell}}}^{T}\right) .
$$

Here $L_{k}(k \geq 0)$ denotes the bidiagonal $k \times(k+1)$ pencil

$$
L_{k}:=\left[\begin{array}{cccc}
z & -1 & & \\
& \ddots & \ddots & \\
& & z & -1
\end{array}\right]
$$

$N_{f}$ and $M_{\infty}$ are two square matrices in real Jordan form, with $M_{\infty}$ nilpotent.

The regular part of $z M-N$ is defined by the regular pencil $\operatorname{diag}\left(I_{n_{\infty}}, N_{f}\right)-$ $z \operatorname{diag}\left(M_{\infty}, I_{n_{f}}\right)$. The finite generalized eigenvalues (and their multiplicities) of the pencil $z M-N$ are the eigenvalues (and their multiplicities) of the matrix $N_{f} . z M-N$ has an infinite generalized eigenvalue if $n_{\infty}>0$, and its multiplicities are defined as the corresponding multiplicities of the eigenvalue at 0 of the nilpotent matrix $N_{\infty}$. A generalized eigenvalue $\lambda$ is called stable if $\lambda \in \mathbb{D}$, and antistable if $\lambda \notin \mathbb{D}$. According to this definition, an infinite generalized eigenvalue is antistable.

The singular part of the pencil is defined by the right and left singular Kronecker structure as follows. The $\epsilon_{i} \times\left(\epsilon_{i}+1\right)$ blocks $L_{\epsilon_{i}},\left(i=1, \ldots, \nu_{r}\right)$, are the right elementary Kronecker blocks, and $\epsilon_{i} \geq 0$ are called the right Kronecker indices. The $\left(\eta_{j}+1\right) \times \eta_{j}$ blocks $L_{\eta_{j}}^{T},\left(j=1, \ldots, \nu_{\ell}\right)$, are the left elementary Kronecker blocks, and $\eta_{j} \geq 0$ are called the left Kronecker indices. Notice that $\epsilon_{i}$ and $\eta_{j}$ can be zero. 
For our future developments we need an equivalent form of (2.1). Let $n_{s}$ and $n_{a}$ be the number (multiplicity counted) of finite stable and antistable generalized eigenvalues, respectively. By using a mere permutation of rows and columns of (2.1) it is straightforward to see that for any pencil $z M-N$ there exist two invertible matrices $Q$ and $Z$ such that

$Q(z M-N) Z=\operatorname{diag}\left(\left[\begin{array}{ll}z I_{n_{r}}-A_{r} & -B\end{array}\right], z I_{n_{s}}-J_{s}, z I_{n_{a}}-J_{a}, z M_{\infty}-I_{n_{\infty}},\left[\begin{array}{c}z I_{n_{\ell}}-A_{\ell} \\ -C\end{array}\right]\right)$

where $\left(A_{r}, B\right)$ is a controllable pair, with $B \in \mathbb{R}^{n_{r} \times \nu_{r}}, J_{s} \in \mathbb{R}^{n_{s} \times n_{s}}$ and $J_{a} \in \mathbb{R}^{n_{a} \times n_{a}}$ are two matrices containing the finite spectrum inside and outside $\mathbb{D}$, respectively, $M_{\infty} \in \mathbb{R}^{n_{\infty} \times n_{\infty}}$ is nilpotent, and $\left(C, A_{\ell}\right)$ is an observable pair, with $C \in \mathbb{R}^{\nu_{\ell} \times n_{\ell}}$.

Finally, we recall the definition of proper deflating subspaces [34, 21] which are used to replace for the case of singular pencils the invariant subspaces of matrices and deflating subspaces of regular pencils.

Definition 1. Let $z M-N$, with $M, N \in \mathbb{R}^{m \times n}$, be a fixed pencil. A subspace $\mathcal{V} \subset \mathbb{R}^{n}$ of dimension $\rho$ is called right deflating if for an arbitrary basis matrix $V \in$ $\mathbb{R}^{n \times \rho}$ of $\mathcal{V}$ there is an $\rho \times \rho$ matrix $S$ such that

$$
N V=M V S .
$$

The right deflating subspace $\mathcal{V}$ is called proper if $M V$ has full column rank, and it is called stable if $\Lambda(S) \subset \mathbb{D}$. Since we are interested only in right deflating subspaces, further on we will simply omit this adjective.

REMARK 1. Deflating subspaces of regular pencils have been introduced in [44] and extended to singular pencils in [47] under the name of reducing subspaces. However, in the case of singular pencils proper deflating subspaces differ somehow from reducing subspaces and these differences have been investigated in [36].

3. Popov systems: definitions, associated objects, and equivalence. We introduce here the basic setting of our approach to the discrete-time nonsymmetric Riccati theory. The central concept is the Popov-system. With a fixed Popov system we associate several mathematical objects: the discrete-time nonsymmetric algebraic Riccati system and a particular version of it called the discrete-time nonsymmetric algebraic Riccati equation, the Popov function and the Popov-system pencil which are nothing else but the transfer function and system pencil of the Popov-system, respectively. Finally, we will introduce an equivalence transformation on Popovsystems that plays an important part in alternative time- and frequency-domain characterizations of stabilizing solutions. All developments are considered in discretetime and provide the natural extension of the classical symmetric Riccati theory [21] to the nonsymmetric case.

Consider the discrete-time Popov-system,

$$
\left\{\begin{array}{rlll}
\sigma x & =A_{1} x & & +B_{1} u, \\
\lambda & =Q x+A_{2}{ }^{\prime} \sigma \lambda & +L_{1} u, \\
\nu & =L_{2}{ }^{\prime} x+B_{2}{ }^{\prime} \sigma \lambda & +R u,
\end{array}\right.
$$

where $\sigma$ is the shift operator acting on a vector valued sequence $w=(w(k))_{k \in \mathbb{Z}}$ as $(\sigma w)(k)=w(k+1)$. Here the state $x=(x(k))_{k \in \mathbb{N}}$, the costate (or dual) state $\lambda=(\lambda(k))_{k \in \mathbb{N}}$, the input $u=(u(k))_{k \in \mathbb{N}}$ and the output $\nu=(\nu(k))_{k \in \mathbb{N}}$ are sequences in $\mathbb{R}^{n}, \mathbb{R}^{\ell}, \mathbb{R}^{m}$ and $\mathbb{R}^{m}$, respectively, and all the intervening matrices in (3.1) have appropriate dimensions. Let $x_{0}$ be the initial condition on the state, i.e., $x(0)=x_{0}$. 
Alternatively, to denote the Popov-system (3.1) we use the following abbreviated notation $\mathcal{P}=\left(A_{1}, B_{1}, A_{2}, B_{2}, Q, L_{1}, L_{2}{ }^{\prime}, R\right)$. Let $\mathcal{P}^{*}=\left(A_{2}, B_{2}, A_{1}, B_{1}, Q^{\prime}, L_{2}, L_{1}{ }^{\prime}, R^{\prime}\right)$ denote the dual Popov-system associated with (3.1).

3.1. DTNARS. Let $\mathcal{P}$ be the Popov system (3.1). For any matrix $X \in \mathbb{C}^{\ell \times n}$, associate with $\mathcal{P}$ the matrix

$$
\mathcal{D}_{\mathcal{P}}(X)=\left[\begin{array}{cc}
A_{2}{ }^{\prime} X A_{1}-X+Q & A_{2}{ }^{\prime} X B_{1}+L_{1} \\
B_{2}{ }^{\prime} X A_{1}+L_{2}{ }^{\prime} & R+B_{2}{ }^{\prime} X B_{1}
\end{array}\right] .
$$

DeFinition 2. The system of equations

$$
\mathcal{D}_{\mathcal{P}}\left(X_{1}\right)\left[\begin{array}{l}
I_{n} \\
F_{1}
\end{array}\right]=0
$$

in the unknowns $X_{1} \in \mathbb{C}^{\ell \times n}$ and $F_{1} \in \mathbb{C}^{m \times n}$ is called the right discrete-time nonsymmetric algebraic Riccati system (DTNARS) associated with $\mathcal{P}$. A solution $\left(X_{1}, F_{1}\right)$ to (3.3) is called right stabilizing if $\Lambda\left(A_{1}+B_{1} F_{1}\right) \subset \mathbb{D}$.

Similarly, the system of equations

$$
\left[\begin{array}{ll}
I_{n} & F_{2}{ }^{\prime}
\end{array}\right] \mathcal{D}_{\mathcal{P}}\left(X_{2}\right)=0
$$

in the unknowns $X_{2} \in \mathbb{C}^{\ell \times n}$ and $F_{2} \in \mathbb{C}^{m \times \ell}$ is called the left DTNARS associated with $\mathcal{P}$. A solution $\left(X_{2}, F_{2}\right)$ satisfying (3.4) is said to be left stabilizing if

$$
\Lambda\left(A_{2}+B_{2} F_{2}\right) \subset \mathbb{D} .
$$

The following result which is a variation of [24, prop 6.9] shows the connection between right and left stabilizing solutions.

Proposition 1. Assume $\left(X_{1}, F_{1}\right)$ and $\left(X_{2}, F_{2}\right)$ are right and left stabilizing solutions to the DTNARS (3.3) and (3.4), respectively. Then $X_{1}$ and $X_{2}$ are unique and

$$
X_{1}=X_{2}
$$

Proof. We show first (3.6). From the definition of the right stabilizing solution, one obtains

$$
\begin{array}{r}
A_{2}{ }^{\prime} X_{1} A_{1}-X_{1}+A_{2}{ }^{\prime} X_{1} B_{1} F_{1}+L_{1} F_{1}+Q=0 \\
B_{2}{ }^{\prime} X_{1} A_{1}+L_{2}{ }^{\prime}+\left(R+B_{2}{ }^{\prime} X_{1} B_{1}\right) F_{1}=0
\end{array}
$$

Let $S_{1}=A_{1}+B_{1} F_{1}$ and rewrite the equation (3.7) as

$$
A_{2}{ }^{\prime} X_{1} S_{1}-X_{1}+L_{1} F_{1}+Q=0 .
$$

By premultiplying equality (3.8) by ${F_{2}}^{\prime}$, one obtains

$$
F_{2}{ }^{\prime} B_{2}{ }^{\prime} X_{1} A_{1}+F_{2}{ }^{\prime} L_{2}{ }^{\prime}+F_{2}{ }^{\prime}\left(R+B_{2}{ }^{\prime} X_{1} B_{1}\right) F_{1}=0 .
$$

Add now these two last equations to obtain, by setting $S_{2}=A_{2}+B_{2} F_{2}$

$$
S_{2}{ }^{\prime} X_{1} S_{1}-X_{1}+L_{1} F_{1}+F_{2}{ }^{\prime} L_{2}{ }^{\prime}+F_{2}{ }^{\prime} R F_{1}+Q=0 .
$$


Using the similar technique for the left stabilizing Riccati solution, one gets

$$
S_{2}{ }^{\prime} X_{2} S_{1}-X_{2}+L_{1} F_{1}+F_{2}{ }^{\prime} L_{2}{ }^{\prime}+F_{2}{ }^{\prime} R F_{1}+Q=0 .
$$

Substracting equality (3.11) from equality (3.12) leads to

$$
S_{2}{ }^{\prime}\left(X_{1}-X_{2}\right) S_{1}-\left(X_{1}-X_{2}\right)=0 .
$$

Since $S_{1}$ and $S_{2}$ are both stable by definition, this Stein equation has a unique solution, which must be $X_{1}-X_{2}=0$.

The unicity follows by repeating the arguments for pairs of an arbitrary right stabilizing solution $X_{1}$ and a fixed left stabilizing solution $X_{2 f}$, and for pairs of an arbitrary left stabilizing solution $X_{2}$ and a fixed right stabilizing solution $X_{1 f}$.

REMARK 2. The transpose of the left DTNARS (3.4) coincides with the right DTNARS associated with the dual Popov-system $\mathcal{P}^{*}$ in the unknowns $\left(X^{\prime}, F_{2}\right)$. This observation allows us to consider in the sequel only the right DTNARS.

3.2. DTNARE. Let $\mathcal{P}$ be the Popov system (3.1). Suppose that $\left(X, F_{1}\right)$ is a solution to the right DTNARS (3.3) and in addition $\left(R+B_{2}{ }^{\prime} X B_{1}\right)$ is invertible. Then $F_{1}$ could be made explicit as

$$
F_{1}=-\left(R+B_{2}{ }^{\prime} X B_{1}\right)^{-1}\left(B_{2}{ }^{\prime} X A_{1}+L_{2}{ }^{\prime}\right)
$$

Substituting the expression (3.14) into the first equation of the DTNARS (3.3), one obtains the discrete-time nonsymmetric algebraic Riccati equation (DTNARE) associated with the Popov-system $\mathcal{P}$

$$
A_{2}{ }^{\prime} X A_{1}-X+Q-\left(L_{1}+A_{2}{ }^{\prime} X B_{1}\right)\left(R+B_{2}{ }^{\prime} X B_{1}\right)^{-1}\left(B_{2}{ }^{\prime} X A_{1}+L_{2}{ }^{\prime}\right)=0 .
$$

Definition 3. A solution $X$ to DTNARE (3.15) is called stabilizing if $A_{1}+B_{1} F_{1}$ is stable, for $F_{1}$ defined in (3.14). In this case, $F_{1}$ is called the (right) stabilizing Riccati feedback.

The form (3.15) of DTNARE is directly related to the DTNARS (3.3) by the Schur complement. However, if both $R$ and $\left(I+B_{1} R^{-1} B_{2}{ }^{\prime} X\right)$ are invertible we get the following more familiar equivalent form which occurs widely in the literature:

$$
\begin{aligned}
-X+Q-L_{1} R^{-1} L_{2}{ }^{\prime}+ & \left(A_{2}{ }^{\prime}-L_{1} R^{-1} B_{2}{ }^{\prime}\right) \\
& \times X\left(I+B_{1} R^{-1} B_{2}{ }^{\prime} X\right)^{-1}\left(A_{1}-B_{1} R^{-1} L_{2}{ }^{\prime}\right)=0 .
\end{aligned}
$$

The transformation between these two forms is obvious. Indeed, using (3.14) we get successively

$$
\begin{aligned}
F_{1} & =-\left(R+B_{2}{ }^{\prime} X B_{1}\right)^{-1}\left(B_{2}{ }^{\prime} X A_{1}+L_{2}{ }^{\prime}\right) \\
& =-R^{-1}\left(L_{2}{ }^{\prime}+B_{2}{ }^{\prime} X\left(I+B_{1} R^{-1} B_{2}{ }^{\prime} X\right)^{-1}\left(A_{1}-B_{1} R^{-1} L_{2}^{\prime}\right)\right),
\end{aligned}
$$

and

$$
\begin{aligned}
A_{1}+B_{1} F_{1} & =A_{1}-B_{1}\left(R+B_{2}{ }^{\prime} X B_{1}\right)^{-1}\left(B_{2}{ }^{\prime} X A_{1}+L_{2}{ }^{\prime}\right) \\
& =\left(I+B_{1} R^{-1} B_{2}{ }^{\prime} X\right)^{-1}\left(A_{1}-B_{1} R^{-1} L_{2}{ }^{\prime}\right) .
\end{aligned}
$$

Further, rewriting (3.16) as

$$
-X+Q+A_{2}^{\prime} X\left(A_{1}+B_{1} F_{1}\right)+L_{1} F_{1}
$$

and replacing $F_{1}$ and $A+B_{1} F_{1}$ from (3.17) and (3.18), respectively, we get precisely (3.16). As it will be shown later on, the choice between these forms is guided by the application, in particular by the value of the matrices $L_{1}$ and $L_{2}$. 
3.3. The Popov function, the Popov-system pencil, and an equivalence relation. Let $\mathcal{P}$ be the Popov-system (3.1). The transfer matrix from $u$ to $\nu$,

$$
\boldsymbol{\Pi}_{\mathcal{P}}(z)=\left[\begin{array}{ll}
B_{2}{ }^{\prime}\left(z^{-1} I-A_{2}{ }^{\prime}\right)^{-1} & I
\end{array}\right]\left[\begin{array}{cc}
Q & L_{1} \\
L_{2}^{\prime} & R
\end{array}\right]\left[\begin{array}{c}
\left(z I-A_{1}\right)^{-1} B_{1} \\
I
\end{array}\right]
$$

is called the (nonsymmetric) Popov function.

Let $\mathcal{P}$ be a Popov-system. The system (transmission) pencil $z M_{\mathcal{P}}-N_{\mathcal{P}}$ associated with $\mathcal{P}$ is called the Popov-system pencil, where

$$
M_{\mathcal{P}}=\left[\begin{array}{ccc}
I_{n} & 0 & 0 \\
0 & -A_{2}{ }^{\prime} & 0 \\
0 & -B_{2}{ }^{\prime} & 0
\end{array}\right], \quad N_{\mathcal{P}}=\left[\begin{array}{ccc}
A_{1} & 0 & B_{1} \\
Q & -I_{\ell} & L_{1} \\
L_{2}{ }^{\prime} & 0 & R
\end{array}\right]
$$

Since the $z M_{\mathcal{P}}-N_{\mathcal{P}}$ is the transmission pencil and $\boldsymbol{\Pi}_{\mathcal{P}}(z)$ the transfer matrix of the Popov-system, it follows that the Popov-system pencil is regular if and only if the Popov function is square and has full rank.

We introduce now an equivalence transformation for Popov systems.

Definition 4. Two Popov-systems

$$
\mathcal{P}=\left(A_{1}, B_{1}, A_{2}, B_{2}, Q, L_{1}, L_{2}{ }^{\prime}, R\right), \quad \widetilde{\mathcal{P}}=\left(\widetilde{A}_{1}, \widetilde{B}_{1}, \widetilde{A}_{2}, \widetilde{B}_{2}, \widetilde{Q}, \widetilde{L}_{1}, \widetilde{L}_{2}^{\prime}, \widetilde{R}\right)
$$

are called $\left(X, F_{1}, F_{2}\right)$-equivalent if there are matrices $X \in \mathbb{C}^{\ell \times n}, F_{1} \in \mathbb{C}^{m \times n}$ and $F_{2} \in \mathbb{C}^{m \times \ell}$ such that the following conditions hold

$$
\begin{aligned}
\widetilde{A}_{1} & =A_{1}+B_{1} F_{1}, \\
\widetilde{A}_{2} & =A_{2}+B_{2} F_{2}, \\
\widetilde{B}_{1} & =B_{1}, \\
\widetilde{B}_{2} & =B_{2}, \\
\widetilde{L}_{1} & =L_{1}+A_{2}{ }^{\prime} X B_{1}+F_{2}{ }^{\prime}\left(R+B_{2}^{\prime} X B_{1}\right), \\
\widetilde{L}_{2}^{\prime} & =L_{2}{ }^{\prime}+B_{2}{ }^{\prime} X\left(A_{1}+B_{1} F_{1}\right)+R F_{1}, \\
\widetilde{Q} & =Q+F_{2}{ }^{\prime} R F_{1}+L_{1} F_{1}+F_{2}{ }^{\prime} L_{2}{ }^{\prime}+\left(A_{2}+B_{2} F_{2}\right)^{\prime} X\left(A_{1}+B_{1} F_{1}\right)-X, \\
\widetilde{R} & =R+B_{2}{ }^{\prime} X B_{1} .
\end{aligned}
$$

It is straightforward to check that the relation defined above is indeed an equivalence relation fulfilling the corresponding axioms.

The next result gives the relation between the above mathematical objects associated with two equivalent Popov-systems.

Proposition 2. Let

$$
\mathcal{P}=\left(A_{1}, B_{1}, A_{2}, B_{2}, Q, L_{1}, L_{2}{ }^{\prime}, R\right), \widetilde{\mathcal{P}}=\left(\widetilde{A_{1}}, \widetilde{B_{1}}, \widetilde{A_{2}}, \widetilde{B_{2}}, \widetilde{Q}, \widetilde{L_{1}},{\widetilde{L_{2}}}^{\prime}, \widetilde{R}\right),
$$

be two $\left(X, F_{1}, F_{2}\right)$-equivalent Popov systems.

1. $\left(X_{s}, F_{s}\right)$ is a (stabilizing) solution to the DTNARS associated with $\mathcal{P}$ if and only if $\left(X_{s}-X, F_{s}-F_{1}\right)$ is a (stabilizing) solution to the DTNARS associated with $\widetilde{\mathcal{P}}$.

2. For $z \in \mathbb{C} \backslash\left\{\Lambda\left(A_{1}\right) \cup \Lambda\left(A_{2}\right) \cup \Lambda\left(\widetilde{A_{1}}\right) \cup \Lambda\left(\widetilde{A_{2}}\right)\right\}$ we have

$$
\boldsymbol{\Pi}_{\mathcal{P}}(z)=\mathbf{S}_{F_{2}}^{*}(z) \boldsymbol{\Pi}_{\widetilde{\mathcal{P}}}(z) \mathbf{S}_{F_{1}}(z)
$$


where

$$
\mathbf{S}_{F_{i}}(z):=I-F_{i}\left(z I-A_{i}\right)^{-1} B_{i}, \quad i=1,2 .
$$

Moreover, if $\left(X_{s 1}, F_{s 1}\right),\left(X_{s 2}, F_{s 2}\right)$ are right and left solutions to the right and left DTNARS associated with the Popov-system $\mathcal{P}$, respectively, and $X_{s 1}=$ $X_{s 2}=: X_{s}$, then

$$
\Pi_{\mathcal{P}}(z)=\mathbf{S}_{F_{s 2}}^{*}(z)\left(R+B_{2}^{\prime} X_{s} B_{1}\right) \mathbf{S}_{F_{s 1}}(z),
$$

where $\mathbf{S}_{F_{s i}}(z)$ is (3.30) written for $F_{i}=F_{s i}, i=1,2$. (The identity (3.31) is the nonsymmetric spectral factorization identity and (3.30) are the spectral factors.)

3. The matrix pencils $z M_{\mathcal{P}}-N_{\mathcal{P}}$ and $z M_{\widetilde{\mathcal{P}}}-N_{\widetilde{\mathcal{P}}}$ are strictly equivalent.

Proof. 1. Let $X_{\Delta}:=X_{s}-X, F_{\Delta}:=F_{s}-F_{1}$. We show first that

$$
D_{\mathcal{P}}\left(X_{s}\right)\left[\begin{array}{c}
I \\
F_{s}
\end{array}\right]=0 \quad \Leftrightarrow \quad D_{\widetilde{\mathcal{P}}}\left(X_{\Delta}\right)\left[\begin{array}{c}
I \\
F_{\Delta}
\end{array}\right]=0
$$

which will prove the relation between the solutions of the DTNARSs. Indeed, we have

$$
\begin{aligned}
& {\left[\begin{array}{cc}
A_{2}{ }^{\prime} X_{s} A_{1}-X_{s}+Q & A_{2}{ }^{\prime} X_{s} B_{1}+L_{1} \\
B_{2}^{\prime} X_{s} A_{1}+L_{2}{ }^{\prime} & R+B_{2}{ }^{\prime} X_{s} B_{1}
\end{array}\right]\left[\begin{array}{c}
I \\
F_{s}
\end{array}\right]=0} \\
& \Leftrightarrow\left[\begin{array}{cc}
A_{2}{ }^{\prime} X_{\Delta} A_{1}-X_{\Delta}+Q+A_{2}^{\prime} X A_{1}-X & A_{2}{ }^{\prime} X_{\Delta} B_{1}+L_{1}+A_{2}{ }^{\prime} X B_{1} \\
B_{2}{ }^{\prime} X_{\Delta} A_{1}+L_{2}{ }^{\prime}+B_{2}{ }^{\prime} X A_{1} & R+B_{2}{ }^{\prime} X_{\Delta} B_{1}+B_{2}{ }^{\prime} X B_{1}
\end{array}\right]\left[\begin{array}{c}
I \\
F_{s}
\end{array}\right]=0 \\
& \Leftrightarrow\left[\begin{array}{cc}
I & F_{2}{ }^{T} \\
0 & I
\end{array}\right]\left[\begin{array}{cc}
A_{2}{ }^{\prime} X_{\Delta} A_{1}-X_{\Delta}+Q+A_{2}^{\prime} X A_{1}-X & A_{2}{ }^{\prime} X_{\Delta} B_{1}+L_{1}+A_{2}{ }^{\prime} X B_{1} \\
B_{2}{ }^{\prime} X_{\Delta} A_{1}+L_{2}{ }^{\prime}+B_{2}{ }^{\prime} X A_{1} & R+B_{2}{ }^{\prime} X_{\Delta} B_{1}+B_{2}{ }^{\prime} X B_{1}
\end{array}\right] \\
& \times\left[\begin{array}{cc}
I & 0 \\
F_{1} & I
\end{array}\right]\left[\begin{array}{cc}
I & 0 \\
-F_{1} & I
\end{array}\right]\left[\begin{array}{c}
I \\
F_{s}
\end{array}\right]=0 \\
& \Leftrightarrow\left[\begin{array}{cc}
\widetilde{A}_{2}^{\prime} X_{\Delta} \widetilde{A}_{1}-X_{\Delta}+\widetilde{Q} & \widetilde{A}_{2}^{\prime} X_{\Delta} \widetilde{B}_{1}+\widetilde{L}_{1} \\
\widetilde{B}_{2}^{\prime} X_{\Delta} \widetilde{A}_{1}+\widetilde{L}_{2}^{\prime} & \widetilde{R}+\widetilde{B}_{2}^{\prime} X_{\Delta} \widetilde{B}_{1}
\end{array}\right]\left[\begin{array}{c}
I \\
F_{\Delta}
\end{array}\right]=0 .
\end{aligned}
$$

The fact that $\left(X_{s}, F_{s}\right)$ is a stabilizing solution to the DTNARS associated with $\mathcal{P}$ if and only if $\left(X_{\Delta}, F_{\Delta}\right)$ is a stabilizing solution to the DTNARS associated with $\widetilde{\mathcal{P}}$ is obvious.

2. For $i=1,2$, we have successively

$$
\begin{aligned}
& \left(z I-\widetilde{A}_{i}\right)^{-1} B_{i} \mathbf{S}_{F_{i}}(z) \\
& =\left(z I-A_{i}-B_{i} F_{i}\right)^{-1} B_{i}\left[I-F_{i}\left(z I-A_{i}\right)^{-1} B_{i}\right] \\
& =\left(z I-A_{i}-B_{i} F_{i}\right)^{-1}\left[B_{i}-B_{i} F\left(z I-A_{i}\right)^{-1} B_{i}\right] \\
& =\left(z I-A_{i}-B_{i} F_{i}\right)^{-1}\left[I-B_{i} F_{i}\left(z I-A_{i}\right)^{-1}\right] B_{i} \\
& =\left(z I-A_{i}-B_{i} F_{i}\right)^{-1}\left(z I-A_{i}-B_{i} F_{i}\right)\left(z I-A_{i}\right)^{-1} B_{i} \\
& =\left(z I-A_{i}\right)^{-1} B_{i} .
\end{aligned}
$$


Using this we get further

$$
\left[\begin{array}{c}
\left(z I-\widetilde{A}_{i}\right)^{-1} B_{i} \\
I
\end{array}\right] \mathbf{S}_{F_{i}}(z)=\left[\begin{array}{c}
\left(z I-A_{i}\right)^{-1} B_{i} \\
I-F_{i}\left(z I-A_{i}\right)^{-1} B_{i}
\end{array}\right]=\left[\begin{array}{cc}
I & 0 \\
-F_{i} & I
\end{array}\right]\left[\begin{array}{c}
\left(z I-A_{i}\right)^{-1} B_{i} \\
I
\end{array}\right] .
$$

Based on this identity, we readily obtain

$$
\begin{aligned}
\mathbf{S}_{F_{2}}^{*}(z) \boldsymbol{\Pi}_{\widetilde{\mathcal{P}}}(z) \mathbf{S}_{F_{1}}(z)= & {\left[B_{2}^{\prime}\left(\frac{1}{z} I-A_{2}^{\prime}\right)^{-1} I\right]\left[\begin{array}{cc}
I & -F_{2}^{\prime} \\
0 & I
\end{array}\right]\left[\begin{array}{cc}
\widetilde{Q} & \widetilde{L_{1}} \\
\widetilde{L_{2}} & \widetilde{R}
\end{array}\right] } \\
& \times\left[\begin{array}{cc}
I & 0 \\
-F_{1} & I
\end{array}\right]\left[\begin{array}{c}
\left(z I-A_{1}\right)^{-1} B_{1} \\
I
\end{array}\right] .
\end{aligned}
$$

A straightforward computation gives

$$
\left[\begin{array}{cc}
I & -F_{2}^{\prime} \\
0 & I
\end{array}\right]\left[\begin{array}{cc}
\widetilde{Q} & \widetilde{L}_{1} \\
\widetilde{L}_{2}^{\prime} & \widetilde{R}
\end{array}\right]\left[\begin{array}{cc}
I & 0 \\
-F_{1} & I
\end{array}\right]=\left[\begin{array}{cc}
Q & L_{1} \\
L_{2}^{\prime} & R
\end{array}\right]+\left[\begin{array}{cc}
A_{2}^{\prime} X A_{1}-X & A_{2}^{\prime} X B_{1} \\
B_{2}^{\prime} X A_{1} & B_{2}^{\prime} X B_{1}
\end{array}\right]
$$

Combining the last two identities, we conclude that (3.29) is proved, if we show that

$$
\left[B_{2}^{\prime}\left(\frac{1}{z} I-A_{2}^{\prime}\right)^{-1} \quad I\right]\left[\begin{array}{cc}
A_{2}^{\prime} X A_{1}-X & A_{2}^{\prime} X B_{1} \\
B_{2}^{\prime} X A_{1} & B_{2}^{\prime} X B_{1}
\end{array}\right]\left[\begin{array}{c}
\left(z I-A_{1}\right)^{-1} B_{1} \\
I
\end{array}\right]=0 .
$$

To this end, let

$$
\left[\begin{array}{cc}
A_{2}^{\prime} X A_{1}-X & A_{2}^{\prime} X B_{1} \\
B_{2}^{\prime} X A_{1} & B_{2}^{\prime} X B_{1}
\end{array}\right]=\left[\begin{array}{c}
A_{2}^{\prime} \\
B_{2}^{\prime}
\end{array}\right] X\left[\begin{array}{ll}
A_{1} & B_{1}
\end{array}\right]-\left[\begin{array}{cc}
X & 0 \\
0 & 0
\end{array}\right] .
$$

We have

$$
\left[\begin{array}{ll}
A_{1} & B_{1}
\end{array}\right]\left[\begin{array}{c}
\left(z I-A_{1}\right)^{-1} B_{1} \\
I
\end{array}\right]=\left[A_{1}\left(z I-A_{1}\right)^{-1}+I\right] B_{1}=z\left(z I-A_{1}\right)^{-1} B_{1}
$$

and

$$
\left[B_{2}^{\prime}\left(\frac{1}{z} I-A_{2}^{\prime}\right)^{-1} \quad I\right]\left[\begin{array}{l}
A_{2}^{\prime} \\
B_{2}^{\prime}
\end{array}\right]=\frac{1}{z} B_{2}^{\prime}\left(\frac{1}{z} I-A_{2}^{\prime}\right)^{-1} .
$$

With (3.33), (3.34) and (3.35) the left-hand side of (3.32) becomes

$$
B_{2}^{\prime}\left(\frac{1}{z} I-A_{2}^{\prime}\right)^{-1} X\left(z I-A_{1}\right)^{-1} B_{1}-B_{2}^{\prime}\left(\frac{1}{z} I-A_{2}^{\prime}\right)^{-1} X\left(z I-A_{1}\right)^{-1} B_{1}=0
$$

and thus (3.32) is proved.

If $\left(X_{s}, F_{s 1}\right)$ and $\left(X_{s}, F_{s 2}\right)$ are a right and left solution of the DTNARS associated with $\mathcal{P}$, respectively, then it is easily checked that the $\left(X_{s}, F_{s 1}, F_{s 2}\right)$-equivalent of $\mathcal{P}$ has $\widetilde{Q}=0, \widetilde{L}_{1}=0$, and $\widetilde{L}_{2}=0$. Hence, the Popov function associated with $\widetilde{\mathcal{P}}$ is $\Pi_{\widetilde{\mathcal{P}}}(z)=R+B_{2}^{\prime} X_{s} B_{1}$, and (3.31) follows as a consequence of (3.29).

3. If $\mathcal{P}$ and $\widetilde{\mathcal{P}}$ are two $\left(X, F_{1}, F_{2}\right)$-equivalent Popov-systems, then the matrices $X, F_{1}, F_{2}$ verify the relations (3.21)-(3.28). A direct check shows that

$$
z M_{\mathcal{P}}-N_{\mathcal{P}}=U\left(z M_{\widetilde{\mathcal{P}}}-N_{\widetilde{\mathcal{P}}}\right) V
$$


where $U$ and $V$ defined by

$$
U=\left[\begin{array}{ccc}
I & 0 & 0 \\
-A_{2}{ }^{\prime} X & I & -F_{2}^{\prime} \\
-B_{2}^{\prime} X & 0 & I
\end{array}\right], V=\left[\begin{array}{ccc}
I & 0 & 0 \\
-X & I & 0 \\
-F_{1} & 0 & I
\end{array}\right]
$$

are clearly invertible and their inverses are given by

$$
U^{-1}=\left[\begin{array}{ccc}
I & 0 & 0 \\
\widetilde{A}_{2}^{\prime} X & I & F_{2}^{\prime} \\
\widetilde{B}_{2}^{\prime} X & 0 & I
\end{array}\right], V^{-1}=\left[\begin{array}{ccc}
I & 0 & 0 \\
X & I & 0 \\
F_{1} & 0 & I
\end{array}\right] .
$$

Combining Proposition 1 and 2 it follows that the spectral factorization identity (3.31) holds if the DTNARS associated with $\mathcal{P}$ has a right and a left stabilizing solution.

4. Main result. In this section we present the main result of the paper which establishes a one-to-one correspondence between stabilizing solutions of the DTNARS and stable deflating subspaces of the associated Popov-system pencil. We introduce first a definition.

Definition 5. Let $\mathcal{P}$ be a Popov-system. An n-dimensional deflating subspace $\mathcal{V}$ of the Popov-system pencil $z M_{\mathcal{P}}-N_{\mathcal{P}}$ is called disconjugate provided it has a basis matrix

$$
V=\left[\begin{array}{l}
V_{1} \\
V_{2} \\
V_{3}
\end{array}\right] \begin{array}{ll}
\} & n \\
\} & \ell \\
& m
\end{array}
$$

with $V_{1}$ invertible.

Notice that disconjugacy of a deflating subspace is independent of the choice of the basis matrix for $V$. Moreover, if the deflating subspace is disconjugate then automatically is proper as can be seen from

$$
M_{\mathcal{P}} V=\left[\begin{array}{c}
V_{1} \\
-A_{2}{ }^{\prime} V_{2} \\
-B_{2}{ }^{\prime} V_{2}
\end{array}\right] .
$$

This last property will be used in the sequel to compute numerically different solutions to DTNARS. The next theorem contains the central contribution of the paper and is a rich extension of known results to allow working without any restrictive assumptions on the matrix coefficients.

Theorem 1. Let $\mathcal{P}$ be the Popov-system (3.1). The right DTNARS associated with $\mathcal{P}$ given in (3.3) has a stabilizing solution $\left(X, F_{1}\right)$ if and only if the Popov-system pencil $z M_{\mathcal{P}}-N_{\mathcal{P}}$ given in (3.20) has a stable disconjugate deflating subspace $\mathcal{V}$ of dimension $n$. Provided $V \in \mathbb{C}^{(n+\ell+m) \times n}$ is a basis matrix of $\mathcal{V}$ partitioned as in (4.1), the stabilizing solution is given by

$$
X=V_{2} V_{1}^{-1}, \quad F_{1}=V_{3} V_{1}^{-1} .
$$


Proof. [Sufficiency.] If $z M_{\mathcal{P}}-N_{\mathcal{P}}$ has a stable deflating subspace of dimension $n$ which admits a basis matrix of the form (4.1), then (2.3) shows that there is a stable matrix $S$ such that

$$
\begin{aligned}
A_{1} V_{1}+B_{1} V_{3} & =V_{1} S, \\
Q V_{1}-V_{2}+L_{1} V_{3} & =-A_{2}{ }^{\prime} V_{2} S, \\
L_{2}{ }^{\prime} V_{1}+R V_{3} & =-B_{2}{ }^{\prime} V_{2} S .
\end{aligned}
$$

Since $\mathcal{V}$ is disconjugate, $V_{1}$ is invertible, and multiplying these equations to the right with $V_{1}^{-1}$ leads to

$$
\begin{aligned}
A_{1}+B_{1} V_{3} V_{1}{ }^{-1} & =V_{1} S V_{1}{ }^{-1}, \\
Q-V_{2} V_{1}{ }^{-1}+L_{1} V_{3} V_{1}{ }^{-1} & =-A_{2}{ }^{\prime} V_{2} V_{1}{ }^{-1} V_{1} S V_{1}{ }^{-1}, \\
L_{2}{ }^{\prime}+R V_{3} V_{1}{ }^{-1} & =-B_{2}{ }^{\prime} V_{2} V_{1}{ }^{-1} V_{1} S V_{1}{ }^{-1} .
\end{aligned}
$$

Denoting $X:=V_{2} V_{1}^{-1}, F_{1}:=V_{3} V_{1}^{-1}$ and $\widetilde{S}:=V_{1} S V_{1}^{-1}$, we obtain

$$
\begin{aligned}
A_{1}+B_{1} F_{1} & =\widetilde{S}, \\
Q-X+L_{1} F_{1} & =-A_{2}{ }^{\prime} X \widetilde{S}, \\
L_{2}{ }^{\prime}+R F_{1} & =-B_{2}{ }^{\prime} X \widetilde{S} .
\end{aligned}
$$

Substituting $\widetilde{S}$ from (4.6) in (4.7) and (4.8), we deduce that $X$ and $F_{1}$ fulfill

$$
\begin{aligned}
Q-X+A_{2}{ }^{\prime} X A_{1}+L_{1} F_{1}+A_{2}{ }^{\prime} X B_{1} F_{1} & =0, \\
L_{2}{ }^{\prime}+B_{2}{ }^{\prime} X A_{1}+R F_{1}+B_{2}{ }^{\prime} X B_{1} F_{1} & =0,
\end{aligned}
$$

i.e., $\left(X, F_{1}\right)$ is a solution to the right DTNARS (3.3). Furthermore $S$ being stable, $\widetilde{S}=A_{1}+B_{1} F_{1}$ is also stable which proves that $\left(X, F_{1}\right)$ is a stabilizing solution to the DTNARS (3.3).

[Necessity.] If $\left(X, F_{1}\right)$ is a right stabilizing solution to the DTNARS then (3.3) can be rewritten as

$$
N_{\mathcal{P}}\left[\begin{array}{c}
I_{n} \\
X \\
F_{1}
\end{array}\right]=M_{\mathcal{P}}\left[\begin{array}{c}
I_{n} \\
X \\
F_{1}
\end{array}\right]\left(A_{1}+B_{1} F_{1}\right) .
$$

Since by assumption $\left(A_{1}+B_{1} F_{1}\right)$ is stable, the space spanned by $\left[\begin{array}{ccc}I_{n} & X^{T} & F_{1}^{T}\end{array}\right]^{T}$ is a stable deflating subspace. The subspace is in addition disconjugate, due to the invertibility of $I_{n}$.

Contrary to alternative approaches available in the literature (see for example [9]), Theorem 1 does not assume any hypothesis on the matrix coefficients of the underlying Popov-system $\mathcal{P}$. In particular, the matrix $R$ could be singular, while $A_{1}$ and $A_{2}$ could be singular and have an arbitrary spectrum (not necessary stable). However, when $R$ is invertible, we have

$$
\begin{gathered}
{\left[\begin{array}{ccc}
I_{n} & 0 & -B_{1} R^{-1} \\
0 & I_{\ell} & -L_{1} R^{-1} \\
0 & 0 & I_{m}
\end{array}\right]\left(z M_{\mathcal{P}}-N_{\mathcal{P}}\right)\left[\begin{array}{ccc}
I_{n} & 0 & 0 \\
0 & I_{\ell} & 0 \\
-R^{-1} L_{2}{ }^{\prime} & 0 & R^{-1}
\end{array}\right]=} \\
\quad z\left[\begin{array}{ccc}
I_{n} & B_{1} R^{-1} B_{2}{ }^{\prime} & 0 \\
0 & -A_{2}{ }^{\prime}+L_{1} R^{-1} B_{2}{ }^{\prime} & 0 \\
0 & -B_{2}{ }^{\prime} & 0
\end{array}\right]-\left[\begin{array}{ccc}
A_{1}-B_{1} R^{-1} L_{2}{ }^{\prime} & 0 & 0 \\
Q-L_{1} R^{-1} L_{2}{ }^{\prime} & -I_{\ell} & 0 \\
0 & 0 & I_{m}
\end{array}\right]
\end{gathered}
$$


which shows that the Popov-system pencil is regular and can be replaced for computational purposes with the pencil

$$
\widehat{M}_{\mathcal{P}}=\left[\begin{array}{cc}
I_{n} & B_{1} R^{-1} B_{2}{ }^{\prime} \\
0 & -\left(A_{2}{ }^{\prime}-L_{1} R^{-1} B_{2}{ }^{\prime}\right)
\end{array}\right], \quad \widehat{N}_{\mathcal{P}}=\left[\begin{array}{cc}
A_{1}-B_{1} R^{-1} L_{2}{ }^{\prime} & 0 \\
Q-L_{1} R^{-1} L_{2}{ }^{\prime} & -I_{\ell}
\end{array}\right]
$$

The generalized eigenvalues of $z M_{\mathcal{P}}-N_{\mathcal{P}}$ are those of $z \widehat{M}_{\mathcal{P}}-\widehat{N}_{\mathcal{P}}$ plus $m$ infinite ones. If in addition $\left(A_{2}{ }^{\prime}-L_{1} R^{-1} B_{2}{ }^{\prime}\right)$ is invertible, the deflating subspace of (4.11) can be replaced by the classical concept of invariant subspace of the matrix $\widehat{M}_{\mathcal{P}}^{-1} \widehat{N}_{\mathcal{P}}$ (or, respectively, if $\left(A_{1}-B_{1} R^{-1} L_{2}{ }^{\prime}\right)$ is invertible of the matrix $\widehat{N}_{\mathcal{P}}^{-1} \widehat{M}_{\mathcal{P}}[10]$ ).

REMARK 3. Let $\mathbb{C}=\mathbb{C}_{g} \cup \mathbb{C}_{b}$ be a disjoint partition of the complex plane in two symmetric sets ( $a$ good one and a bad one). Similar results establishing a one-toone correspondence between solutions $\left(X, F_{1}\right)$ to the DTNARS with $\Lambda\left(A_{1}+B_{1} F_{1}\right) \subset$ $\mathbb{C}_{g}$, and disconjugate deflating subspaces of the Popov-system pencil fulfilling (2.3) with $\Lambda(S) \subset \mathbb{C}_{g}$, can be obtained by simply replacing in the above definitions and derivations the open unit disk with $\mathbb{C}_{g}$, while the adjective stable pertains now to $\mathbb{C}_{g}$. Moreover, as will become clear in the next section, also the numerical algorithms can go through. In particular, one obtains a complete characterization of semi-stabilizing solutions to the DTNARS for which $\mathbb{C}_{g}=\overline{\mathbb{D}}$.

5. Computation of the stabilizing solutions to the DTNARS. In this section we present a numerically-sound approach for checking the solvability conditions of and computing the stabilizing solutions to the DTNARS.

Theorem 1 establishes a one-to-one correspondence between stabilizing solutions of DTNARSs and stable disconjugate (and implicitly proper) deflating subspaces of dimension $n$ of the Popov-system pencil. Although the theorem gives a necessary and sufficient criterion for the solvability of the DTNARS, it is not very useful for the numerical computation of the solution since, in general, there may be none or many $n$-dimensional proper stable deflating subspaces. Therefore we need a tool to decide if any such subspace exists and, if so, to give a way to construct these subspaces and implicitly the solutions to DTNARS. The following theorem offers a criterion for ensuring the existence of a proper stable deflating subspace of dimension $n$ of a general pencil.

Theorem 2. Let $z M-N$ be an arbitrary pencil. Denote by $n_{r}$ the sum of the right Kronecker indices and by $n_{s}$ the number (multiplicity counted) of stable generalized eigenvalues of $z M-N$. Then $z M-N$ has a proper stable deflating subspace of dimension $n$ if and only if $n_{r}+n_{s} \geq n$.

Proof. [Necessity.] Let $\mathcal{V}$ be a proper stable deflating subspace of dimension $n$ for $z M-N$ and let $V \in \mathbb{C}^{(n+\ell+m) \times n}$ be a basis matrix for it. Following Definition 1, there exists a square matrix $S$, with $\Lambda(S) \subset \mathbb{D}$, such that

$$
N V=M V S,
$$

with $M V$ of full column rank. Let $Q$ and $Z$ be two invertible matrices that bring the pencil to the form (2.2). Then (5.1) can be rewritten as

$$
Q N Z Z^{-1} V=Q M Z Z^{-1} V S
$$


where

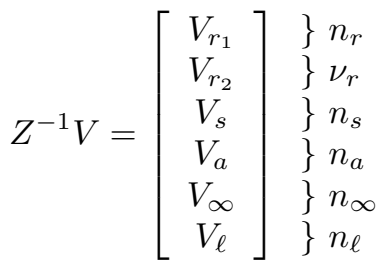

has been partitioned conformably with (2.2). Here $\nu_{r}, n_{a}$, and $n_{\infty}$ are the number of right Kronecker blocks, the number of finite antistable generalized eigenvalues, and the number of infinite generalized eigenvalues, respectively.

From (5.2) we extract

$$
\begin{aligned}
J_{a} V_{a} & =V_{a} S, \\
V_{\infty} & =M_{\infty} V_{\infty} S, \\
A_{\ell} V_{\ell} & =V_{\ell} S, \\
C V_{\ell} & =0 .
\end{aligned}
$$

Since $\left(C, A_{\ell}\right)$ is observable there exists $K$ such that $\Lambda\left(A_{\ell}+K C\right) \subset \mathbb{C} \backslash \mathbb{D}$. Hence the two last equations yield

$$
\left(A_{\ell}+K C\right) V_{\ell}=V_{\ell} S .
$$

Since $\Lambda(S) \cap \Lambda\left(A_{\ell}+K C\right)=\Lambda(S) \cap \Lambda\left(J_{a}\right)=\emptyset$, we infer from (5.4) and (5.8) that $V_{a}=0$ and $V_{\ell}=0$. From (5.5) we show now that $V_{\infty}=0$. Indeed, since $M_{\infty}$ is nilpotent let $k \in \mathbb{N}$ be its nilpotency index. Then $M_{\infty}^{k}=0$ and multiplying to the left (5.5) with $M_{\infty}^{(k-1)}$, it follows that $M_{\infty}^{(k-1)} V_{\infty}=0$. Multiplying to the left (5.5) with $M_{\infty}^{(k-2)}$, we get $M_{\infty}^{(k-2)} V_{\infty}=0$. Iterating this, we end with $M_{\infty} V_{\infty}=0$, and (5.5) gives $V_{\infty}=0$. Hence we showed

$$
Q M V=\left[\begin{array}{c}
V_{r_{1}} \\
V_{s} \\
0_{t \times n}
\end{array}\right],
$$

$\left(t:=n_{a}+n_{\infty}+n_{\ell}+\nu_{\ell}\right)$. Since $\mathcal{V}$ is a proper deflating subspace of dimension $n$, and $Q$ is invertible, $M V$ has full column rank and therefore $\left[\begin{array}{c}V_{r_{1}} \\ V_{s}\end{array}\right]$ has also full column rank. It follows $\operatorname{dim} \mathcal{V}=n \leq\left(n_{r}+n_{s}\right)$.

[Sufficiency.] Assume that $\left(n_{r}+n_{s}\right) \geq n$. We show, constructively, that there exists a proper stable deflating subspace of dimension $n$. We consider separately the following two cases: $n_{s} \geq n$ and $n_{s}<n$.

If $n_{s} \geq n$, it suffices to select $n$ eigenvalues among the $n_{s}$ stable ones. Define

$$
V=Z\left[\begin{array}{c}
0_{\left(n_{r}+\nu_{r}\right) \times n} \\
I_{n} \\
0_{\alpha \times n}
\end{array}\right]
$$

with $\alpha=n_{s}-n+n_{a}+n_{\infty}+n_{\ell}$. A direct check shows that $V$ satisfies (5.2) and has rank equal to $n$. Since $M V=Q^{-1}\left[\begin{array}{c}0_{n_{r} \times n} \\ I_{n} \\ 0_{\alpha \times n}\end{array}\right]$ has full column rank, we conclude that $\operatorname{Im}(V)$ is a proper stable deflating subspace of dimension $n$. 
If $n_{s}<n$, since the pair $\left(A_{r}, B\right)$ is controllable, there exist matrices $F \in \mathbb{R}^{\nu_{r} \times n_{r}}$, $A_{c 1} \in \mathbb{R}^{\left(n-n_{s}\right) \times\left(n-n_{s}\right)}, A_{c 2} \in \mathbb{R}^{\left(n_{r}+n_{s}-n\right) \times\left(n_{r}+n_{s}-n\right)}$ and $T \in \mathbb{R}^{n_{r} \times n_{r}}$, with $T$ invertible, such that

$$
\left(A_{r}+B F\right)=T\left[\begin{array}{cc}
A_{c 1} & 0 \\
0 & A_{c 2}
\end{array}\right] T^{-1}
$$

with $\Lambda\left(A_{c 1}\right) \subset \mathbb{D}$. Denote by $T_{\left(n-n_{s}\right)} \in \mathbb{R}^{n_{r} \times\left(n-n_{s}\right)}$ the matrix built from the first $\left(n-n_{s}\right)$ columns of $T$. Define

$$
V=Z\left[\begin{array}{cc}
I_{n_{r}} & 0 \\
F & 0 \\
0 & I_{n_{s}} \\
0 & 0_{\alpha \times n_{s}}
\end{array}\right]\left[\begin{array}{cc}
T_{\left(n-n_{s}\right)} & 0 \\
0 & I_{n_{s}}
\end{array}\right]=\left[\begin{array}{cc}
T_{\left(n-n_{s}\right)} & 0 \\
F T_{\left(n-n_{s}\right)} & 0 \\
0_{n_{s} \times\left(n-n_{s}\right)} & I_{n_{s}} \\
0_{\alpha \times\left(n-n_{s}\right)} & 0_{\alpha \times n_{s}}
\end{array}\right]
$$

with $\alpha:=n-n_{s}+n_{a}+n_{\infty}+n_{\ell}$. A direct check shows that (5.2) holds with $S=\left[\begin{array}{cc}A_{c 1} & 0 \\ 0 & J_{s}\end{array}\right], \Lambda(S) \subset \mathbb{D}$, and $V$ and $M V$ have both full column rank. Hence we conclude again that the subspace spanned by $V$ is a proper stable deflating subspace of dimension $n$. This ends the whole proof.

We comment on the possibility of using the above result to compute the stabilizing solution to the DTNARS associated with $\mathcal{P}$. The maximal dimension of a proper stable deflating subspace of $z M_{\mathcal{P}}-N_{\mathcal{P}}$ is $\left(n_{r}+n_{s}\right)$. However, even stable proper deflating subspaces of maximal dimension are in general non-unique. Though, at least when $n_{r}+n_{s}=n$ this non-uniqueness does not affect the possibility to find the solution to the DTNARS associated with $\mathcal{P}$, as shown in the following proposition.

Proposition 3. Let $\mathcal{P}$ be a Popov-system (3.1) and $\left(z M_{\mathcal{P}}-N_{\mathcal{P}}\right)$ the associated pencil. If $\left(z M_{\mathcal{P}}-N_{\mathcal{P}}\right)$ has a maximal proper stable deflating subspace of dimension $n$ which is disconjugate then each maximal proper stable deflating subspace is disconjugate.

Proof. We show first that if $V$ is a basis matrix for a maximal proper stable deflating subspace $\mathcal{V}$ then $\operatorname{Im}\left(M_{\mathcal{P}} V\right)$ is independent of the particular choice of $\mathcal{V}$. Let $Q$ and $Z$ be the invertible matrices that bring the pencil to the form (2.2). From (5.9) it follows that the matrix $\left[\begin{array}{cc}V_{r 1}^{T} & V_{s}^{T}\end{array}\right]^{T}$ has full column rank and, since the dimension of $\mathcal{V}$ is $n=n_{r}+n_{s}$, it is also square and, therefore, invertible. Now

$$
\operatorname{Im}\left(M_{\mathcal{P}} V\right)=\operatorname{Im}\left(Q^{-1}\left[\begin{array}{c}
V_{r 1} \\
V_{a} \\
0
\end{array}\right]\right)=\operatorname{Im}\left(Q^{-1}\left[\begin{array}{c}
I_{n_{r}+n_{s}} \\
0
\end{array}\right]\right),
$$

which is clearly independent of the particular choice of $\mathcal{V}$.

Let $\mathcal{V}$ and $\overline{\mathcal{V}}$ be two maximal stable subspaces with basis matrices

$$
V=\left[\begin{array}{lll}
V_{1}^{T} & V_{2}^{T} & V_{3}^{T}
\end{array}\right]^{T}, \quad \bar{V}=\left[\begin{array}{ccc}
\bar{V}_{1}^{T} & \bar{V}_{2}^{T} & \bar{V}_{3}^{T}
\end{array}\right]^{T},
$$

respectively. Then

$$
M_{\mathcal{P}} V=\left[\begin{array}{c}
V_{1} \\
-A_{2}^{\prime} V_{2} \\
-B_{2}^{\prime} V_{2}
\end{array}\right], \quad M_{\mathcal{P}} \bar{V}=\left[\begin{array}{c}
\bar{V}_{1} \\
-A_{2}^{\prime} \bar{V}_{2} \\
-B_{2}^{\prime} \bar{V}_{2}
\end{array}\right]
$$


have full column rank and as proved before their images coincide. Hence there exists an invertible $G$ such that

$$
\left[\begin{array}{c}
V_{1} \\
-A_{2}^{\prime} V_{2} \\
-B_{2}^{\prime} V_{2}
\end{array}\right]=\left[\begin{array}{c}
\bar{V}_{1} \\
-A_{2}^{\prime} \bar{V}_{2} \\
-B_{2}^{\prime} \bar{V}_{2}
\end{array}\right] G
$$

Thus if $V_{1}$ is invertible, then $\overline{V_{1}}$ is too.

The previous results show that depending on the matrix coefficients three distinct cases may occur:

- If $n_{r}+n_{s}<n$, the DTNARS has no stabilizing solution;

- If $n_{r}+n_{s}=n$ then the DTNARS may have a stabilizing solution and its existence can be decided by computing any maximal proper stable deflating subspace of the Popov-system pencil and checking its disconjugacy. Moreover, under the mild assumption that the pair $\left(A_{2}, B_{2}\right)$ is controllable at $z=0$ it can be easily seen from (5.13) that $V_{2}=\bar{V}_{2} G$. Indeed, let $F$ be a feedback matrix such that $A_{2}+B_{2} F$ is invertible. Then from (5.13) we get

$$
\left[\begin{array}{c}
-\left(A_{2}+B_{2} F\right)^{\prime} \\
-B_{2}^{\prime}
\end{array}\right] V_{2}=\left[\begin{array}{c}
-\left(A_{2}+B_{2} F\right)^{\prime} \\
-B_{2}^{\prime}
\end{array}\right] \bar{V}_{2} G
$$

from where $V_{2}=\bar{V}_{2} G$. Therefore the stabilizing solution $X=V_{2} V_{1}^{-1}=$ $\bar{V}_{2} \bar{V}_{1}^{-1}$ is unique in this case and can be computed from any proper stable deflating subspace of maximal dimension $n$.

- If $n_{r}+n_{s}>n$, the DTNARS may have none, one, or many distinct stabilizing solutions. However, even in this case the existence of solutions may be decided by computing a maximal stable deflating subspace of $z M_{\mathcal{P}}-N_{\mathcal{P}}$ as explained further. In view of Theorem 1 we are interested only in disconjugate stable deflating subspaces $\mathcal{V}$ of dimension $n$ (which are automatically proper). Any such subspace fulfills $\mathcal{V} \subset \mathcal{X}$, and $M_{\mathcal{P}} \mathcal{V} \subset \mathcal{Y}$, where $(\mathcal{X}, \mathcal{Y})$ is the (unique) pair of reducing subspaces corresponding to the stable eigenvalues of $z M_{\mathcal{P}}-N_{\mathcal{P}}$. Moreover, $\mathcal{X}$ is precisely the maximal stable deflating subspace (see [36]) and all it remains is to decide if $\mathcal{X}$ contains any proper subspace and to compute these subspaces. More details are given in the numerical algorithm presented below.

It is apparent from the previous discussion that the main step in computing the solution of a DTNARS is to find the maximal stable deflating subspace and, if possible, to select from it a proper deflating subspaces of dimension $n$. The algorithm given below follows quite closely the proof of Theorem 2, except that it avoids the use of the Kronecker form, which is a poor tool in numerical computations.

Algorithm for computing stabilizing solutions to DTNARS. Let $\mathcal{P}$ be a Popov-system (3.3) and $z M_{\mathcal{P}}-N_{\mathcal{P}}$ the associated pencil.

Step 1. Compute unitary matrices $Q$ and $Z$ that bring $z M_{\mathcal{P}}-N_{\mathcal{P}}$ to the generalized Schur form

$$
Q\left(z M_{\mathcal{P}}-N_{\mathcal{P}}\right) Z=\left[\begin{array}{cccc}
z M_{\epsilon}-N_{\epsilon} & \star & \star & \star \\
0 & z M_{s}-N_{s} & \star & \star \\
0 & 0 & z M_{a}-N_{a} & \star \\
0 & 0 & 0 & z M_{\eta}-N_{\eta}
\end{array}\right],
$$

(see for example (2.5) in [3]), where $z M_{s}-N_{s}$ (with dimensions $n_{s} \times n_{s}$ ) and $z M_{a}-N_{a}$ (with dimensions $n_{a} \times n_{a}$ ) are regular pencils containing the stable and antistable 
generalized eigenvalues, respectively, while the singular right and left parts of the pencil are determined by $z M_{\epsilon}-N_{\epsilon}$ (with dimensions $n_{r} \times\left(n_{r}+\nu_{r}\right)$ ) and $z M_{\eta}-N_{\eta}$ (with dimensions $\left.\left(n_{\ell}+\nu_{\ell}\right) \times n_{\ell}\right)$, respectively. In particular, both $M_{\epsilon}$ and $N_{\epsilon}$ have full row rank and outline the following partition $z M_{\epsilon}-N_{\epsilon}=z\left[0 \mid M_{\epsilon 2}\right]-\left[N_{\epsilon 1} \mid N_{\epsilon 2}\right]$, where $M_{\epsilon 2}$ is square, block upper triangular and nonsingular, and the pair $\left(M_{\epsilon 2}^{-1} N_{\epsilon 2}\right.$, $\left.M_{\epsilon 2}^{-1} N_{\epsilon 1}\right)$ is controllable.

Step 2. If $n_{r}+n_{s}<n$ the DTNARS has no solution (as there is no proper deflating subspace of dimension $n$ - see Theorem 2). Otherwise, let $K$ be a feedback matrix such that $z M_{\epsilon 2}-N_{\epsilon 2}-N_{\epsilon 1} K$ has solely stable generalized eigenvalues. The existence of the feedback matrix $K$ follows from the controllability of the pair $\left(M_{\epsilon 2}^{-1} N_{\epsilon 2}\right.$, $\left.M_{\epsilon 2}^{-1} N_{\epsilon 1}\right)$. The feedback $K$ can be computed either by explicitly inverting the matrix $M_{\epsilon 2}$ and applying an eigenvalue assignment algorithm or, better, without inverting $M_{\epsilon 2}$ by using the algorithm in [34] based on the generalized Schur form of the regular pencil $z M_{\epsilon 2}-N_{\epsilon 2}$. The effect of the feedback on the whole pencil $z M_{\mathcal{P}}-N_{\mathcal{P}}$ is that of a strict equivalence. Update $Q$ and $Z$ and get (by reusing notation)

$$
Q\left(z M_{\mathcal{P}}-N_{\mathcal{P}}\right) Z=\left[\begin{array}{cccc}
-N_{\epsilon 1} & z M_{\epsilon 2}-N_{\epsilon 1} K-N_{\epsilon 2} & z M_{\epsilon s}-N_{\epsilon s} & \star \\
0 & 0 & z M_{s}-N_{s} & \star \\
0 & 0 & 0 & \star \\
0 & 0 & 0 & \star
\end{array}\right] .
$$

Denoting the first $n_{\max }:=n_{r}+\nu_{r}+n_{s}$ columns of $Z$ by $V_{1}$ we conclude that $M_{\mathcal{P}} V_{1} S=$ $N_{\mathcal{P}} V_{1}$ holds with

$$
S:=\left[\begin{array}{ccc}
S_{a} & 0 & 0 \\
M_{\epsilon 2}^{-1} N_{\epsilon 1} & M_{\epsilon 2}^{-1}\left(N_{\epsilon 1} K+N_{\epsilon 2}\right) & M_{\epsilon 2}^{-1}\left(N_{\epsilon s}-M_{\epsilon s} M_{s}^{-1} N_{s}\right) \\
0 & 0 & M_{s}^{-1} N_{s}
\end{array}\right],
$$

where $S_{a}$ is an arbitrary $\nu_{r} \times \nu_{r}$ matrix with $\Lambda\left(S_{a}\right) \subset \mathbb{D}$. Clearly, $\Lambda(S) \subset \mathbb{D}$ and therefore $\mathcal{V}_{1}=\operatorname{Im}\left(V_{1}\right)$ is the (unique) maximal stable deflating subspace which coincides with $\mathcal{X}$, where $(\mathcal{X}, \mathcal{Y})$ is the maximal stable reducing pair.

Step 3. Let $V_{1}=\left[\begin{array}{lll}V_{11}^{T} & V_{21}^{T} & V_{31}^{T}\end{array}\right]^{T}$ be partitioned as $V$ in (2.3).

If $\operatorname{rank}\left(V_{11}\right)<n$ then the DTNARS has no stabilizing solution (as there is no disconjugate deflating subspace of dimension $n$ ).

If $\operatorname{rank}\left(V_{11}\right) \geq n$ then the DTNARS may have none, one, a finite number, or an uncountable set of stabilizing solutions corresponding to various possible choices of $n$-dimensional disconjugate stable deflating subspaces included in $\mathcal{V}_{1}$. Each stable deflating subspace included in $\mathcal{V}_{1}$ is in correspondence with an $S$-invariant subspace. Provided the matrix $S$ has eigenvalues with geometric multiplicity 1, all stable deflating subspaces of dimension $n$ can be selected by bringing the matrix $S$ to ordered Schur forms with different arrangements of the first $n$ eigenvalues on the main diagonal. In this case the number $N$ of all $n$-dimensional stable deflating subspaces, which is an upper bound for the number of all stabilizing solutions of the DTNARS, fulfils $N \leq\left(\begin{array}{c}n_{\max } \\ n\end{array}\right)$. Each such subspace which is in addition disconjugate corresponds to a stabilizing solution to the DTNARS. If the matrix $S$ has eigenvalues with geometric multiplicity greater than one, then there exists always an uncountable set of $n$-dimensional stable deflating subspaces (corresponding to the uncountable set of $n$-dimensional $S$-invariant subspaces) and in this case the DTNARS may also have an uncountable set of stabilizing solutions. However, $\Lambda(S)=\Lambda\left(S_{a}\right) \cup \Lambda\left(M_{\epsilon 2}^{-1}\left(N_{\epsilon 1} K+N_{\epsilon 2}\right)\right) \cup \Lambda\left(M_{s}^{-1} N_{s}\right)$ and at least the matrix $S_{a}$ and 
$K$ can always be chosen such that the $\Lambda\left(S_{a}\right) \cup \Lambda\left(M_{\epsilon 2}^{-1}\left(N_{\epsilon 1} K+N_{\epsilon 2}\right)\right)$ has distinct elements. In particular, by selecting sets of eigenvalues such that any eigenvalue in the set appears together with its complex-conjugated pair the resulting solutions to the DTNARS are real.

Step 4. Let $\bar{V}_{1}=\left[\begin{array}{ccc}\bar{V}_{11}^{T} & \bar{V}_{21}^{T} & \bar{V}_{31}^{T}\end{array}\right]^{T}$ be a basis matrix of an $n$-dimensional stable disconjugate subspace, partitioned conformably to $V$ in (2.3). Compute the corresponding stabilizing solution to the DTNARS as $X=\bar{V}_{21} \bar{V}_{11}^{-1}, F=\bar{V}_{31} \bar{V}_{11}^{-1}$.

We comment briefly on numerical aspects of the proposed algorithm: operation count and numerical stability. The computational burden lies with Step 1 in which the pencil is brought to the generalized Schur form. The computation of this form requires $\mathcal{O}\left(m^{2} n\right)$ operations for the $m \times n$ pencil $z M_{\mathcal{P}}-N_{\mathcal{P}}$, provided the efficient algorithm in [3] is used. Moreover, the algorithm in [3] uses solely unitary transformations leading to numerical backward stability. Reordering of eigenvalues or generalized eigenvalues is potentially needed in each of the first three steps of the algorithm (provided at Step 2 the generalized Schur type algorithm of [34] is used). For reordering eigenvalues of an $n \times n$ matrix the $\mathcal{O}\left(n^{3}\right)$ backward stable algorithm in [13] is recommended while for generalized eigenvalues the backward stable algorithm [46] can be used leading to a similar operation count of $\mathcal{O}\left(n^{3}\right)$ for a regular $n \times n$ pencil. Besides these backward stable unitary transformations, two nonunitary ones are needed: at Step 2 (the stabilizing feedback matrix $K$ ) and the final formulas from Step 4. Although they do not affect the overall algorithm complexity, extra care should be taken in order not to compromise the accuracy of the final results. For example, the feedback matrix $K$ should be computed by a robust pole placement algorithm (see Section 6 of [38]) while the explicit inversion of the matrix $\bar{V}_{11}$ should be avoided and implemented through a reliable algorithm for solving linear systems of equations (see [13]).

6. Application of DTNARE to game theory. In game theory $[33,2]$ one builds mathematical models of multiple users behavior in a competitive environment, where the profit of each participant depends on the choices of the others. Game theory offers essential tools to define and find equilibria by providing sets of strategies which should be followed by different players to reach a suitable compromise. The most prominent family of equilibria are related to Nash [31, 32] strategy.

Equilibria depend on the information structure, more precisely on the set of information available to each player to build its own strategy. With an open-loop information structure - as opposed to a closed-loop one - no player has access to the measured value of the system state. This means that the players are committed to follow an a priori strategy. In a linear-quadratic game with infinite time horizon, the existence of a Nash equilibrium is closely related to the solvability of a set of coupled algebraic Riccati equations which can be reformulated as a single nonsymmetric algebraic Riccati equation $[1,7,6,22,18]$. We show further how these results can be recast by our pencil approach while removing the restrictive invertibility hypotheses present in the literature.

Consider a two-player linear quadratic discrete-time game defined by the linear dynamic

$$
\sigma x=A x+B^{1} u_{1}+B^{2} u_{2}, \quad x(0)=x_{0},
$$

and the infinite-time horizon quadratic criteria

$$
J_{i}\left(u_{1}, u_{2}\right)=\sum_{k \in \mathbb{N}} L_{i}\left(x(k), u_{1}(k), u_{2}(k)\right)
$$


where the instantaneous criteria are

$$
L_{i}\left(x, u_{1}, u_{2}\right)=x^{\prime} Q_{i} x+u_{1}^{\prime} R_{i 1} u_{1}+u_{2}^{\prime} R_{i 2} u_{2} .
$$

Here $A \in \mathbb{R}^{n \times n}, B^{i} \in \mathbb{R}^{n \times m_{i}}, Q_{i}=Q_{i}^{\prime} \in \mathbb{R}^{n \times n}, R_{i j}=R_{i j}^{\prime} \in \mathbb{R}^{m_{i} \times m_{j}}, n$ and $m_{i}$ belong to $\mathbb{N}^{*}, x$ is the state of the game, and $u_{i}$ is the input of player $i(i, j=1,2)$. In general $R_{i i}$ are assumed invertible and the criteria are convex, i.e., $Q_{i} \geq 0, R_{i j} \geq 0$, $(i, j=1,2)$.

DeFinition 6. Define the rational reaction sets of the players

$$
\begin{array}{l|l}
\mathcal{R}_{1}\left(u_{2}\right)=\left\{\widetilde{u}_{1} \in \mathcal{U}_{a d, 1}\right. & \left.J_{1}\left(\widetilde{u}_{1}, u_{2}\right) \leq J_{1}\left(u_{1}, u_{2}\right), \forall u_{1} \in \mathcal{U}_{a d, 1}\right\}, \\
\mathcal{R}_{2}\left(u_{1}\right)=\left\{\widetilde{u}_{2} \in \mathcal{U}_{a d, 2}\right. & \left.J_{2}\left(u_{1}, \widetilde{u}_{2}\right) \leq J_{2}\left(u_{1}, u_{2}\right), \forall u_{2} \in \mathcal{U}_{a d, 2}\right\},
\end{array}
$$

where $\mathcal{U}_{a d, i}$ denotes the set of admissible controls $u_{i}$ for the player $i$. The rational reaction set corresponds to the best reply of the player $i$ against a fixed but arbitrary action of the other.

In the following subsection we study open-loop Nash strategy, and show how the computation of equilibrium points reduces to the solution of DTNAREs and corresponding eigenvalue problems for the associated system-pencil.

6.1. Open-Loop Nash strategy and DTNARE. Nash strategy [31, 32] is a solution concept of a noncooperative game in which there is no hierarchy among the players, each player is assumed to know the equilibrium strategies of the other players, and no player has anything to gain by changing unilaterally only his own strategy. Specifically, if each player has chosen his strategy and no player can benefit by changing his strategy while the other players keep theirs unchanged, then the current set of strategy choices constitute a Nash equilibrium. Control design using Nash strategies has been formalized in $[43,42,17]$.

Definition 7. A pair of controls $\left(u_{1}^{*}, u_{2}^{*}\right)$ is a Nash strategy, if and only if, for all $u_{i} \in \mathcal{U}_{a d, i}$

$$
\begin{aligned}
& J_{1}^{*}=J_{1}\left(u_{1}^{*}, u_{2}^{*}\right) \leq J_{1}\left(u_{1}, u_{2}^{*}\right), \\
& J_{2}^{*}=J_{2}\left(u_{1}^{*}, u_{2}^{*}\right) \leq J_{2}\left(u_{1}^{*}, u_{2}\right),
\end{aligned}
$$

i.e., $u_{1}^{*} \in \mathcal{R}_{1}\left(u_{2}^{*}\right), u_{2}^{*} \in \mathcal{R}_{2}\left(u_{1}^{*}\right)$. The couple $\left(J_{1}^{*}, J_{2}^{*}\right)$ is known as the outcome of the associated Nash equilibrium.

The necessary conditions leading to a Nash equilibrium (see [2]) are

$$
\left\{\begin{aligned}
\sigma x & =A x+B^{1} u_{1}+B^{2} u_{2} \\
\lambda_{1} & =Q_{1} x+A^{\prime} \sigma \lambda_{1} \\
\lambda_{2} & =Q_{2} x+A^{\prime} \sigma \lambda_{2} \\
0 & =\left(B^{1}\right)^{\prime} \sigma \lambda_{1}+R_{11} u_{1} \\
0 & =\left(B^{2}\right)^{\prime} \sigma \lambda_{2}+R_{22} u_{2}
\end{aligned}\right.
$$

where $\left(\lambda_{1}(k)\right)_{k \in \mathbb{N}}$ and $\left(\lambda_{2}(k)\right)_{k \in \mathbb{N}}$ are the dual state (or costate) $\mathbb{R}^{n}$-valued vectors related to the rational reaction set of each player.

To these necessary conditions we associate the Popov-system (3.1) by identifying $x=x, \lambda=\left[\begin{array}{c}\lambda_{1} \\ \lambda_{2}\end{array}\right], \ell=2 n, m=m_{1}+m_{2}$, and setting

$$
A_{1}=A \in \mathbb{R}^{n \times n}, \quad B_{1}=\left[\begin{array}{ll}
B^{1} & B^{2}
\end{array}\right] \in \mathbb{R}^{n \times\left(m_{1}+m_{2}\right)}, \quad L_{1}=0_{\ell \times m},
$$




$$
\begin{gathered}
A_{2}=\left[\begin{array}{cc}
A & 0 \\
0 & A
\end{array}\right] \in \mathbb{R}^{2 n \times 2 n}, \quad B_{2}=\left[\begin{array}{cc}
B^{1} & 0 \\
0 & B^{2}
\end{array}\right] \in \mathbb{R}^{2 n \times\left(m_{1}+m_{2}\right)}, \quad L_{2}=0_{n \times m} \\
Q=\left[\begin{array}{c}
Q_{1} \\
Q_{2}
\end{array}\right] \in \mathbb{R}^{2 n \times n}, \quad R=\left[\begin{array}{cc}
R_{11} & 0 \\
0 & R_{22}
\end{array}\right] \in \mathbb{R}^{\left(m_{1}+m_{2}\right) \times\left(m_{1}+m_{2}\right)}
\end{gathered}
$$

Partition $X=\left[\begin{array}{l}X_{1} \\ X_{2}\end{array}\right]$, where $X_{1}$ and $X_{2}$ are in $\mathbb{R}^{n \times n}$.

If the quadratic criterion is convex (i.e., $R_{11}>0$ and $R_{22}>0$ ) then $R$ is invertible and we can define $S_{i}:=B^{i} R_{i i}^{-1}\left(B^{i}\right)^{\prime},(i=1,2)$. Assuming $\left(I_{n}+S_{1} X_{1}+S_{2} X_{2}\right)$ is invertible the DTNARE (3.16) becomes for the actual data

$$
-\left[\begin{array}{l}
X_{1} \\
X_{2}
\end{array}\right]+\left[\begin{array}{l}
Q^{1} \\
Q^{2}
\end{array}\right]+\left[\begin{array}{cc}
A^{\prime} & 0 \\
0 & A^{\prime}
\end{array}\right]\left[\begin{array}{l}
X_{1} \\
X_{2}
\end{array}\right]\left(I_{n}+\left[\begin{array}{ll}
S_{1} & S_{2}
\end{array}\right]\left[\begin{array}{c}
X_{1} \\
X_{2}
\end{array}\right]\right)^{-1} A=0,
$$

or, more compactly,

$$
\begin{aligned}
& X_{1}=Q_{1}+A^{\prime} X_{1}\left(I_{n}+S_{1} X_{1}+S_{2} X_{2}\right)^{-1} A, \\
& X_{2}=Q_{2}+A^{\prime} X_{2}\left(I_{n}+S_{1} X_{1}+S_{2} X_{2}\right)^{-1} A .
\end{aligned}
$$

This equation is called the open-loop Nash Riccati equation [1]. The controls $\left(u_{1}^{*}, u_{2}^{*}\right)$ are then given by

$$
\left[\begin{array}{c}
u_{1}^{*} \\
u_{2}^{*}
\end{array}\right]=u=F_{1} x=-\left[\begin{array}{c}
R_{11}^{-1}\left(B^{1}\right)^{\prime} X_{1} \\
R_{22}^{-1}\left(B^{2}\right)^{\prime} X_{2}
\end{array}\right]\left(I_{n}+S_{1} X_{1}+S_{2} X_{2}\right)^{-1} A .
$$

By applying the transformation (4.10), the pencil $\left(z M_{\mathcal{P}}-N_{\mathcal{P}}\right)$ associated with $\mathcal{P}$ reduces to $\left(z \widehat{M}_{\mathcal{P}}-\widehat{N}_{\mathcal{P}}\right)$, where

$$
\widehat{M}_{\mathcal{P}}=\left[\begin{array}{ccc}
I_{n} & S_{1} & S_{2} \\
0_{n} & -A^{\prime} & 0_{n} \\
0_{n} & 0_{n} & -A^{\prime}
\end{array}\right] ; \quad \widehat{N}_{\mathcal{P}}=\left[\begin{array}{ccc}
A & 0_{n} & 0_{n} \\
Q_{1} & -I_{n} & 0_{n} \\
Q_{2} & 0_{n} & -I_{n}
\end{array}\right]
$$

Assuming in addition that $A$ is invertible, we recover the characterization matrix introduced in [11],

$$
\widehat{N}_{\mathcal{P}}^{-1} \widehat{M}_{\mathcal{P}}=\left[\begin{array}{ccc}
A^{-1} & A^{-1} S_{1} & A^{-1} S_{2} \\
Q_{1} A^{-1} & A^{\prime}+Q_{1} A^{-1} S_{1} & Q_{1} A^{-1} S_{2} \\
Q_{2} A^{-1} & Q_{2} A^{-1} S_{1} & A^{\prime}+Q_{2} A^{-1} S_{2}
\end{array}\right]
$$

7. Numerical examples. We illustrate the benefits of our approach on some simple but relevant examples, from academic world, that cannot be solved by alternative methods.

Example 1. Before presenting game theoretic examples, we consider a generic example of DTNARS leading to singular pencils, in particular where $A_{1}, A_{2}$ and $R$ are not invertible. The example is defined with the Popov-system:

$$
\begin{aligned}
& A_{1}=[0], \quad A_{2}=\left[\begin{array}{cc}
0 & 0.1 \\
0 & 0
\end{array}\right], \quad B_{1}=\left[\begin{array}{ll}
0 & 0.1
\end{array}\right], \\
& B_{2}=\left[\begin{array}{cc}
0 & 0.1 \\
0 & 0
\end{array}\right], \quad Q=\left[\begin{array}{l}
1 \\
3
\end{array}\right], \quad R=\left[\begin{array}{ll}
0 & 0 \\
1 & 0
\end{array}\right] .
\end{aligned}
$$


The Popov-system pencil $z M_{\mathcal{P}}-N_{\mathcal{P}}$ has the following structural elements: $n_{r}=$ $\nu_{r}=\nu_{\ell}=1, n_{\ell}=n_{f}=0, n_{\infty}=3, n_{s}=n_{a}=0$, and therefore is singular, without finite regular part. Due to the fact that $n_{r}=n=1$, it is possible to hope to obtain a real stabilizing solution for the DTNARS. The Popov-system pencil is strictly equivalent to

$$
\left.Q\left(z M_{\mathcal{P}}-N_{\mathcal{P}}\right) Z=\left[\begin{array}{ccccc}
z & 1 & 0 & 0 & 0 \\
0 & 0 & & & \\
0 & 0 & & \star & \\
0 & 0 & & & \\
0 & 0 & & &
\end{array}\right] \text {, with } Z=\left[\begin{array}{cc}
-0.9901 & 0.0000 \\
-0.9901 & -0.0000 \\
-2.9704 & -0.0990 \\
0 & 0.0990 \\
0.0000 & -9.9015
\end{array}\right] \text { }\right] \text {. }
$$

In order to obtain a closed-loop eigenvalue $\gamma$, the first component of $Z\left[\begin{array}{lll}1 & \gamma & 0_{1 \times 3}\end{array}\right]$ is always invertible and leads to

$$
X=\left[\begin{array}{ll}
1 & 3+0.1 \gamma
\end{array}\right]^{\prime}, \quad F_{1}=\left[\begin{array}{ll}
-0.1 \gamma & 10 \gamma
\end{array}\right]^{\prime}, \quad A_{1}+B_{1} F_{1}=\gamma .
$$

Example 2. In this example we consider a Nash strategy for a linear quadratic game in which the matrices $A$ and $R_{11}$ are both singular. Due to this singularity the characteristic matrix approach [11] does not apply. The Popov-system in (6.9)-(6.11) is defined by

$$
\begin{gathered}
A=\left[\begin{array}{ll}
1 & 1 \\
1 & 1
\end{array}\right], \quad B^{1}=\left[\begin{array}{l}
2 \\
0
\end{array}\right], \quad B^{2}=\left[\begin{array}{l}
1.5 \\
-1
\end{array}\right], \quad Q_{1}=\left[\begin{array}{ll}
1 & 1 \\
1 & 2
\end{array}\right], \quad Q_{2}=\left[\begin{array}{ll}
2 & 1 \\
1 & 1
\end{array}\right], \\
R_{11}=0, \quad R_{12}=0, \quad R_{21}=1, \quad R_{22}=2 .
\end{gathered}
$$

The Popov-system pencil $z M_{\mathcal{P}}-N_{\mathcal{P}}$ has the following structural elements: $n_{r}=n_{\ell}=$ $\nu_{r}=\nu_{\ell}=0, n_{f}=4, n_{\infty}=4, n_{s}=3, n_{a}=1$, and therefore is regular. The stable finite eigenvalues are $\{0,0,0.5561\}$ while the unstable eigenvalue is $\{3.9561\}$. We compute a stable deflating subspace corresponding to the following $n=2$ eigenvalues: $\{0,0.5561\}$. The resulting stable deflating subspace is disconjugate leading to the right stabilizing solution $(X, F)$ to the DTNARE associated with $\mathcal{P}$, where

$$
X=\left[\begin{array}{cc}
0.0000 & 0.0000 \\
0.0000 & 1.0000 \\
-21.7805 & -22.7805 \\
-22.7805 & -22.7805
\end{array}\right], \quad F=\left[\begin{array}{cc}
-1.2219 & -1.2219 \\
2.0000 & 2.0000
\end{array}\right]
$$

Example 3. In this case we consider Nash strategy for the linear quadratic game defined by $n=2$,

$$
\begin{gathered}
A=\left[\begin{array}{ll}
3 & 1 \\
1 & 1
\end{array}\right], \quad B^{1}=\left[\begin{array}{l}
2 \\
0
\end{array}\right], \quad B^{2}=\left[\begin{array}{l}
1.5 \\
-1
\end{array}\right], \quad Q_{1}=\left[\begin{array}{ll}
1 & 1 \\
1 & 2
\end{array}\right], \quad Q_{2}=\left[\begin{array}{ll}
2 & 1 \\
1 & 1
\end{array}\right], \\
R_{11}=1, \quad R_{12}=0, \quad R_{21}=1, \quad R_{22}=1 .
\end{gathered}
$$

For Nash equilibria, the Popov-system pencil $z M_{\mathcal{P}}-N_{\mathcal{P}}$ associated with $\mathcal{P}$ in (6.9)-(6.11) has the following structure: $n_{r}=n_{\ell}=\nu_{r}=\nu_{\ell}=0, n_{f}=6, n_{\infty}=2$, $n_{g}=n_{b}=3$. The stable eigenvalues are $\{0.1530+0.1189 i ; 0.1530-0.1189 i ; 0.4424\}$. To obtain a real stabilizing solution to the DTNARE associated with $\mathcal{P}$, we select pairs 
of complex-conjugated eigenvalues, and get a disconjugate proper deflating subspace and the stabilizing solution

$$
X=\left[\begin{array}{ll}
5.2133 & 3.4401 \\
3.2971 & 3.4486 \\
2.1571 & 0.6319 \\
1.2758 & 1.0167
\end{array}\right], \quad F=\left[\begin{array}{cc}
-1.9162 & -0.9915 \\
0.4242 & 0.4978
\end{array}\right]
$$

8. Conclusions. We have presented a comprehensive theory of the discretetime nonsymmetric algebraic Riccati systems under the most general conditions on the matrix coefficients. We have given necessary and sufficient conditions for the existence of stabilizing solutions and we proved that these conditions can be effectively checked and the solutions computed by a numerically-sound procedure. The results have been applied in game theory to design Nash strategy without the classical invertibility assumptions needed in the literature.

\section{REFERENCES}

[1] H. Abou-Kandil, G. Freiling, V. Ionescu, and G. Jank, Matrix Riccati Equations in Control and Systems Theory, Birkhäuser, 2003.

[2] T. Başar And G. J. Olsder, Dynamic Noncooperative Game Theory, SIAM, 1995.

[3] T. Beelen and P. Van Dooren, An improved algorithm for the computation of kronecker's canonical form of a singular pencil, Linear Algebra and its Applications, 105 (1988), pp. 965 .

[4] D. A. Bini, B. IAnnazzo, And F. Poloni, A fast Newton's method for a nonsymmetric algebraic Riccati equation, SIAM J. Matrix Anal. \& Appl., 30 (2008), pp. 276-290.

[5] S. Bittanti, J. A. Laub, And J-C. Willems, The Riccati Equation, Springer Verlag, 1991.

[6] E. Dockner, S. Jørgensen, N. Van Long, and G. Sorger, Differential games in economics and management science, Cambridge University Press, 2000.

[7] J. C. Engwerda, LQ Dynamic Optimization and Differential Games, Wiley, 2005.

[8] A. Ferrante, M. Pavon, and S. Pinzoni, Asymmetric algebraic Riccati equation: a homeomorphic parametrization of the set of solutions, Linear Algebra and its Applications, 329 (2001), pp. 137-156.

[9] G. Freiling, A survey of nonsymmetric Riccati equations, Linear Algebra and its Applications, 351-352 (2002), pp. 243-270.

[10] G. Freiling And G. Jank, Nonsymmetric matrix Riccati equations, Z. Anal. Anwendungen, 14 (1995), pp. 259-284.

[11] G. Freiling, G. Jank, And H. Abou-Kandil, Discrete-time Riccati equations in open-loop Nash and Stackelberg games, European Journal of Control, 5 (1999), pp. 56-66.

[12] F.R. Gantmacher, Theory of matrices, Tomes I and II, Chelsea, New York, 1959.

[13] G. H. Golub and Ch. F. Van Loan, Matrix Computations, John Hopkins Univ. Press, 1989.

[14] C. H. GuO, A new class of nonsymmetric algebraic Riccati equations, Linear Algebra and its Applications, 426 (2007), pp. 636-649.

[15] C. H. Guo and N. J. Higham, Iterative solution of a nonsymmetric algebraic Riccati equation, SIAM J. Matrix Anal. \& Appl., 29 (2007), pp. 396-412.

[16] C. H. Guo And A. J. Laub, On the iterative solution of a class of nonsymmetric algebraic Riccati equation, SIAM J. Matrix Anal. \& Appl., 22 (2000), pp. 376-391.

[17] Y. C. Ho, Survey paper: Differential games, dynamic optimization and generalized control theory, Journal of Optimization Theory and Applications, 6 (1970), pp. 179-209.

[18] V. Ionescu, Reverse discrete-time Riccati equation and extended Nehari problem, Linear Algebra and its Applications, 236 (1996), pp. 59-94.

[19] V. IOnESCU And C. OARĂ, Generalized discrete-time riccati theory, SIAM J. Control Optim., 34 (1996), pp. 601-619.

[20] V. IOnescu, C. OARĂ, ANd M. Weiss, General matrix pencil techniques for the solution of algebraic Riccati equations : a unified approach, IEEE Transactions on Automatic Control, 42 (1997), pp. 1085-1097.

[21] - Generalized Riccati theory and robust control: a Popov function approach, Chichester, New York, John Wiley, 1999. 
[22] J. JuAnG, Existence of algebraic matrix Riccati equations arising in transport theory, Linear Algebra and its Applications, 230 (1995), pp. 89-100.

[23] E.R. Kalman, Contributions to the theory of optimal control, Bol. Soc. Mat. Mexicana, 2 (1960), pp. 102-119.

[24] D. Kremer, Non-symmetric Riccati theory and noncooperative games, PhD thesis, RWTH Aachen, 2003.

[25] V. KǚERA, A contribution to matrix quadratic equations, IEEE Transactions on Automatic Control, (1972), pp. 344-347.

[26] P. Lancaster and L. Rodman, Algebraic Riccati equations, Oxford science publications, 1995.

[27] A. Laub, The Riccati Equation, Springer-Verlag, 1991, ch. Invariant subspace methods for the numerical solution of Riccati equations, pp. 163-196.

[28] A.J. Laub, R.V. Patel, and P.M. Van Dooren, The Control Handbook, W.S. Levine (Eds.), IEEE Press, 1996, ch. Numerical and computational issues in linear control and system theory, pp. 399-414.

[29] A. J. LAUB, A Schur method for solving the algebraic riccati equation, IEEE Transactions on Automatic Control, 30 (1979), pp. 97-108.

[30] J. MedAnIC, Geometric properties and invariant manifolds of the Riccati equation, IEEE Transactions on Automatic Control, 27 (1982), pp. 670-677.

[31] J.F. NAsh, Equilibrium points in $N$-person games, Proceedings Nat. Acad. Science U.S., 36 (1950), pp. 48-49.

[32] - Noncooperative games, Annals of Mathematics, 54 (1951), pp. 286-296.

[33] J. Von Neumann and O. Morgenstern, Theory of Games and Economic Beharvior, Princeton University press, 1944.

[34] C. OARĂ, Proper deflating subspaces: Properties, algorithms and applications, Numerical Algorithms, 7 (1994), pp. 355-373.

[35] - Stabilizing solution to the reverse discrete-time Riccati equation: A matrix pencil based approach, Linear Algebra and its Applications, 246 (1996), pp. 113-130.

[36] C. OARĂ And P. VAn Dooren, An improved algorithm for the computation of structural invariants of a system pencil and related geometric aspects, Systems and Control Letters, 30 (1997), pp. 39-48.

[37] C. OARĂ And R. Ştefan, Generalized Popov and Riccati theories: from factorizations to equations and back, Revue roumaine des sciences techniques, série Électrotechnique et Énergétique, 48 (2003), pp. 457-487.

[38] R. Patel, A. Laub, and P. Van Dooren, Numerical Linear Algebra Techniques for Systems and Control, IEEE Press, Piscataway NJ, 1993.

[39] V. M. Popov, Hyperstability of Control Systems, Springer-Verlag, 1973.

[40] J. E. PotTer, Matrix quadratic solutions, SIAM J. Appl. Math., 14 (1966), pp. 496-501.

[41] W. T. ReID, Riccati differential equations, Academic Press, New York, 1972.

[42] A. W. Starr And Y. C. Ho, Further properties of nonzero-sum differential games, Journal of Optimization Theory and Applications, 3 (1969), pp. 207-219.

[43] — Nonzero-sum differential games, Journal of Optimization Theory and Applications, 3 (1969), pp. 184-206.

[44] G. Stewart, On the sentitivity of the eigenvalue problem ax $=\lambda b x$, SIAM Num. Anal., 9 (1972), pp. 669-686.

[45] A. A. Stoonvogel And A. Saberi, The discrete algebraic Riccati equation and linear matrix inequality, Linear Algebra and its Applications, 274 (1998), pp. 317-365.

[46] P. VAN Dooren, A generalized eigenvalue approach for solving riccati equations, SIAM J. Sci. Stat. Comput., 2 (1981), pp. 121-135.

[47] — Reducing susbpaces: Definitions, properties and algorithms, in Matrix Pencils, Lecture Notes in Mathematics, B. Kågström and A. Ruhe, eds., vol. 973, Springer-Verlag, Berlin, 1983, pp. 58-73.

[48] J. C. WILLEMS, Least squares stationary optimal control and the algebraic Riccati equation, IEEE Transactions on Automatic Control, 16 (1971), pp. 621-634.

[49] H. K. Wimmer, A parametrization of solutions of the discrete-time algebraic riccati equation based on pairs of opposite unmixed solutions, SIAM J. Control Optim., 44 (2006), pp. 19922005. 